\title{
Respiratory symptoms and ventilatory function in confectionery workers
}

\author{
Eugenija Zuskin, Jadranka Mustajbegovic, E Neil Schachter, Josipa Kern
}

\begin{abstract}
Respiratory symptoms and ventilatory capacity were studied in a group of 288 workers (259 women and 29 men) employed in a confectionery plant. A group of workers (96 women and 31 men) not exposed to confectionery manufacture were also studied as controls. The prevalence of chronic respiratory symptoms was higher in exposed than in control workers, being greatest for confectionery workers exposed to the dust of flour, talc, and starch and the vapours of alcohol. Chronic bronchitis was reported by $7 \%$ of the women and $21 \%$ of the men, and chest tightness was reported by $27 \%$ of women and $66 \%$ of men. There was a high prevalence of acute irritative symptoms during the workshift in all groups of confectionery workers, especially for cough, dyspnoea, burning and dryness of the throat, and eye irritation. For all groups of confectionery workers there were statistically significant across shift reductions in ventilatory capacity, being most pronounced for maximum flow rate at $50 \%$ of the control vital capacity $\left(\mathrm{FEF}_{50}\right.$; range 4.6$13.0 \%$ and at $25 \%$ of the control vital capacity $\left(\mathrm{FEF}_{25} ;\right.$ range $\left.4 \cdot 7-22 \cdot 3 \%\right)$. Preshift values of FEF $_{50}$ and FEF $_{25}$ were significantly lower than predicted values. The data suggest that some workers employed in confectionery plants may develop acute and chronic respiratory symptoms associated with changes in lung function.
\end{abstract}

(Occup Environ Med 1994;51:435-439)

Andrija Stampar School of Public Health, Zagreb, Croatia

E Zuskin

J Mustajbegovic

J Kern

The Mount Sinai School of Medicine, New York, NY, USA

E N Schachter

Correspondence to:

Dr E Neil Schachter

Pulmonary Medicine,

Mount Sinai School of

Meunt Sinai School of

Medicine, 1 Gustave L Levy

Place, PO Box 1232, New

YSA.

Accepted for publication 14 March 1994
Work in confectionery plants involves the use of different foods for making sweets and pastries. As a result, confectioners are often exposed to the dusts of flour, talc, starch, walnut, peanut, cacao, cocoa, chocolate, coffee, spices, cinnamon, and other food products. Although the respiratory problems of confectionery workers have not been extensively studied, related data exist for bakery workers. $^{1-8}$ Symptoms among bakers may occur often as noted by Hartman et al who studied 314 workers in an industrial bakery and found that $74 \%$ had respiratory allergy to flour (rhinitis and/or asthma). ${ }^{9}$

As well as studies of bakery workers findings on sensitivity to specific food products are relevant to the study of confectionery workers. Allergy to nuts and beans is widespread in the general population commonly resulting in upper respiratory symptoms. Multiple allergies to common foods including chocolate, walnut, peanut, and other products have been described by Speer. ${ }^{10}$ Nuts, in particular, have been associated with allergic reactions. Hypersensitivity to pine nuts was reported by Fine ${ }^{11}$ and Nielsen, ${ }^{12}$ to pinon nuts by Arnmentia et $a l,,^{13}$ and to pignolia nuts by Santos and Unger. ${ }^{14}$ Allergic and other untoward reactions of the upper respiratory tract due to peanuts have been described by Fries ${ }^{15}$ and Yunginger et al. ${ }^{16} \mathrm{~A}$ case of asthma due to central American walnut was reported by Bush and Clayton. ${ }^{17}$ Allergy to chocolate and cocoa bean are also well recognised and have been reported by Fries. ${ }^{1819}$ Wutrich $^{20}$ described an allergic reaction after the consumption of malt-containing chocolate drinks and snack products. Asthma and other symptoms in cinnamon workers have been reported after exposure to cinnamon dust. ${ }^{21}$

The current study was designed to examine the respiratory function of workers employed in a confectionery plant exposed to many of these substances.

\section{Workers and methods}

\section{WORKERS}

Our study examined 288 workers (259 women and $29 \mathrm{men}$ ) in one confectionery plant in Zagreb, Croatia. They represented $85 \%$ of all workers in the plant. Additionally a group of control workers (65 women and 31 men) of similar age and smoking habit employed as transport workers in the same plant served as a control for respiratory symptoms and baseline lung function. All subjects were volunteers. Table 1 presents the

Table 1 Demographic data of confectionery and control workers

\begin{tabular}{|c|c|c|c|c|c|}
\hline Group & No & Age (y) & $\begin{array}{l}\text { Height } \\
(\mathrm{cm})\end{array}$ & $\begin{array}{l}\text { Exposure } \\
(y)\end{array}$ & $\begin{array}{l}\text { Smokers } \\
(\%)\end{array}$ \\
\hline \multicolumn{6}{|c|}{ Confectionery: } \\
\hline Women & 259 & $\begin{array}{l}33 \\
(16-57)\end{array}$ & $\begin{array}{l}162 \\
(152-177)\end{array}$ & ${ }_{(1-35)}^{11}$ & 15 \\
\hline Men & 29 & $\begin{array}{l}31 \\
(18-56)\end{array}$ & $\begin{array}{l}174 \\
(158-179)\end{array}$ & $\begin{array}{l}10 \\
(1-28)\end{array}$ & 85 \\
\hline \multicolumn{6}{|l|}{ Control: } \\
\hline Women & 65 & $\begin{array}{l}31 \\
(18-58)\end{array}$ & $\begin{array}{l}161 \\
(151-176)\end{array}$ & $\begin{array}{l}10 \\
(1-34)\end{array}$ & 16 \\
\hline Men & 31 & $\begin{array}{l}36 \\
(18-57)\end{array}$ & $\begin{array}{l}174 \\
(156-180)\end{array}$ & $\begin{array}{c}8 \\
(1-26)\end{array}$ & 87 \\
\hline
\end{tabular}

Data are presented as means (range). 
demographic characteristics of the workers and their controls.

\section{WORK ENVIRONMENT AND PROCESSES}

The studied workers were employed in processing various confectionery products such as biscuits, sweets, chocolate, chewing gum, candied fruits, and snack products. We classified workers by specific exposures into five different groups. Group 1 was exposed to aerosols of flour, sugar, starch, talc, and egg powder; group 2 was exposed to the vapours of ethyl alcohol in preparing candied fruits (such as cherry); group 3 was employed in the processing of nuts, almonds, cocoa, cacao, and chocolate; group 4 processed butter, honey, aromatic oil, yeast, and different food colourings; group 5 packed the confectionery products in a cold room. Workers in this plant occasionally rotated their jobs and moved from one working station to another; these areas were completely separated physically. By and large however, workers remained in one area.

\section{RESPIRATORY SYMPTOMS}

Chronic respiratory symptoms were recorded for confectionery workers and control workers with the British Medical Research Council Committee questionnaire on respiratory symptoms $^{22}$ with additional questions on occupational asthma. ${ }^{23}$ For all workers a detailed occupational history as well as questions about their smoking habit were recorded.

The workers were also asked about acute work related symptoms such as cough, dyspnoea, chest tightness, irritation or dryness of the throat, secretion, dryness or bleeding of the nose, and headache. The following definitions were used:

Chronic cough or phlegm - Cough or phlegm production for at least three months a year; Chronic bronchitis-Cough and phlegm for a minimum of three months a year and for not less than two successive years;

Dyspnoea grade 3-Shortness of breath when walking with other people at an ordinary pace on level ground.

Dyspnoea grade 4 Shortness of breath when walking at their own pace on level ground; Occupational asthma-recurring attacks of dyspnoea, chest tightness, and impairment of pulmonary function of the obstructive type associated with or after the workshift. The diagnosis was confirmed by medical records from the employee health centre. Symptoms began or were exacerbated after the start of employment.

\section{VENTILATORY CAPACITY}

Ventilatory capacity was measured in all confectionery and control workers by recording maximum expiratory flow-volume (MEFV) curves with a portable flow-volume spirometer (Pneumoscreen, Jaeger, Germany). Measurements were performed on the first working day of the week (Monday) after two days away from work. All employees were working on the day shift. The forced vital capacity (FVC), forced expiratory volume in one second $\left(\mathrm{FEV}_{1}\right)$, and maximum flow rates at $50 \%$ and the last $25 \%$ of the control vital capacity $\left(\mathrm{FEF}_{50}, \mathrm{FEF}_{25}\right)$ were measured on these curves. At least three MEFV curves were recorded and the highest value was used as the result of the test; MEFV curves in confectionery workers were measured before and after the workshift. The measured Monday preshift values for ventilatory capacity were compared with the predicted normal values defined by Quanjer ${ }^{24}$ and with the control group. The spirometer was calibrated for volume on a daily basis. Both measurements and calibration were performed in accordance with the American Thoracic Society standards for spirometric measurements. ${ }^{25}$

\section{ENVIRONMENTAL DUST MEASUREMENTS}

Airborne dust samples in the confectionery plant were collected with Hexhlet horizontal two stage samplers during the eight hour work shift. Twenty dust samples were collected in the areas where workers were examined. Dust concentrations were expressed separately as the total and the respirable dust fractions in $\mathrm{mg} / \mathrm{m}^{3}$. Vapours of ethyl alcohol were collected in tubes filled with activated charcoal and subsequently analysed by gas chromatography. Vapour concentrations were expressed as parts per million (ppm).

\section{STATISTICAL ANALYSIS}

The results of ventilatory capacity measurements were analysed by paired $t$ test for matched differences (across shift changes and the comparison of baseline to predicted normal values). The unpaired $t$ test was used for measuring differences between exposed and control workers. The $\chi^{2}$ test or when appropriate Fisher's exact test were used for testing differences in the prevalence of respiratory symptoms. Values of $p<0.05$ were considered statistically significant.

\section{Results}

\section{RESPIRATORY SYMPTOMS}

Table 2 presents the prevalence of chronic respiratory symptoms for workers of all groups (men and women) and their controls. Most of the chronic respiratory symptoms occurred significantly more often in confectionery workers than in control workers.

Table 3 shows the prevalence of chronic respiratory symptoms in the five different occupational groups of confectionery workers compared with control workers. Male confectionery workers were employed exclusively in group one jobs. Chronic phlegm, chronic bronchitis, chest tightness, and nasal catarrh were particularly frequent in group 2 women confectioners exposed to ethyl alcohol compared with the other groups of women confectioners. Occupational asthma was reported by seven $(2 \cdot 7 \%)$ of 259 women confectionery workers. These workers stated that their symptoms of asthma were not present before starting employment in the confectionery plant but developed after working for three to 
Table 2 Prevalence of chronic symptoms for workers of all groups and controls

\begin{tabular}{|c|c|c|c|c|c|c|c|c|c|c|}
\hline Group & $\begin{array}{l}\text { Mean } \\
\operatorname{age}(y)\end{array}$ & $\begin{array}{l}\text { Mean } \\
\text { exposure } \\
\text { (y) }\end{array}$ & $\begin{array}{l}\text { Chronic } \\
\text { cough }\end{array}$ & $\begin{array}{l}\text { Chronic } \\
\text { phlegm }\end{array}$ & $\begin{array}{l}\text { Chronic } \\
\text { bronchitis }\end{array}$ & Dyspnoea & $\begin{array}{l}\text { Occu- } \\
\text { pational } \\
\text { asthma }\end{array}$ & $\begin{array}{l}\text { Chest } \\
\text { tightness }\end{array}$ & $\begin{array}{l}\text { Nasal } \\
\text { catarrh }\end{array}$ & Sinusitis \\
\hline \multicolumn{11}{|l|}{ Women: } \\
\hline $\begin{array}{l}\text { Control } \\
(n=65)\end{array}$ & 31 & 10 & $\begin{array}{l}4 \\
(6 \cdot 2)\end{array}$ & $\begin{array}{l}2 \\
(3 \cdot 1)\end{array}$ & $\begin{array}{l}2 \\
(3 \cdot 1)\end{array}$ & $\begin{array}{l}0 \\
(0)\end{array}$ & $\begin{array}{c}0 \\
(0)\end{array}$ & $\begin{array}{l}0 \\
(0)\end{array}$ & $(1.5)$ & $(1 \cdot 5)$ \\
\hline $\begin{array}{l}\text { Confectionery } \\
(n=29)\end{array}$ & 31 & 10 & $\begin{array}{c}8 \\
(27 \cdot 5) \\
N S\end{array}$ & $\begin{array}{c}6 \\
(20 \cdot 7) \\
\text { NS }\end{array}$ & $\begin{array}{c}6 \\
(20 \cdot 7) \\
\text { NS }\end{array}$ & $\begin{array}{l}8 \\
(27 \cdot 6) \\
\mathrm{p}<0.001\end{array}$ & $\begin{array}{c}0 \\
\text { (0) } \\
\text { NS }\end{array}$ & $\begin{array}{l}19 \\
(65.5) \\
0<0.001\end{array}$ & $\begin{array}{l}7 \\
(24 \cdot 1) \\
\mathrm{p}<0 \cdot 001\end{array}$ & $\begin{array}{l}7 \\
(24 \cdot 1) \\
p<0.001\end{array}$ \\
\hline $\begin{array}{l}\text { Control } \\
(n=31)\end{array}$ & 36 & 8 & $\begin{array}{c}6 \\
(19 \cdot 4)\end{array}$ & $\begin{array}{c}5 \\
(16 \cdot 1)\end{array}$ & $\begin{array}{c}5 \\
(16 \cdot 1)\end{array}$ & $\begin{array}{l}2 \\
(2 \cdot 5)\end{array}$ & $\begin{array}{c}0 \\
(0)\end{array}$ & $\begin{array}{l}0 \\
(0)\end{array}$ & $\begin{array}{l}0 \\
(0)\end{array}$ & $\begin{array}{l}0 \\
(0)\end{array}$ \\
\hline
\end{tabular}

Numbers in parentheses are percentages.

Table 3 Prevalence of chronic respiratory symptoms in confectionery workers (groups 1-5) and in control workers

\begin{tabular}{|c|c|c|c|c|c|c|c|c|c|c|c|c|}
\hline Group & Sex & No & $\begin{array}{l}\text { Mean } \\
\operatorname{age}(y)\end{array}$ & $\begin{array}{l}\text { Mean } \\
\text { exposure } \\
(y)\end{array}$ & $\begin{array}{l}\text { Chronic } \\
\text { cough }\end{array}$ & $\begin{array}{l}\text { Chronic } \\
\text { phlegm }\end{array}$ & $\begin{array}{l}\text { Chronic } \\
\text { bronchitis }\end{array}$ & Dyspnoea & $\begin{array}{l}\text { Occu- } \\
\text { pational } \\
\text { asthma }\end{array}$ & $\begin{array}{l}\text { Chest } \\
\text { tightness }\end{array}$ & $\begin{array}{l}\text { Nasal } \\
\text { catarrh }\end{array}$ & Sinusitis \\
\hline 1 & Women & 78 & 32 & 10 & \multirow{8}{*}{$\begin{array}{c}29 \star \star \\
(37 \cdot 2) \\
8 \\
(27 \cdot 5) \\
5 \star \star \\
(26 \cdot 3) \\
2 \star \star \\
(9 \cdot 0) \\
9 \\
(13 \cdot 4) \\
17 \star \star \\
(23 \cdot 3) \\
4 \\
(6 \cdot 2) \\
6 \\
(19 \cdot 4)\end{array}$} & \multirow{8}{*}{$\begin{array}{c}9^{\star} \\
(11 \cdot 5) \\
6 \\
(20 \cdot 7) \\
5^{\star \star} \\
(26 \cdot 3) \\
2 \\
(9 \cdot 0) \\
3 \\
(4 \cdot 5) \\
5 \\
(6 \cdot 8) \\
2 \\
(3 \cdot 1) \\
5 \\
(16 \cdot 1)\end{array}$} & \multirow{8}{*}{$\begin{array}{c}4 \\
(5 \cdot 1) \\
6^{\star \star} \\
(20 \cdot 7) \\
5^{\star \star} \\
(26 \cdot 3) \\
2 \\
(9 \cdot 0) \\
3 \\
(4 \cdot 5) \\
5 \\
(6 \cdot 8) \\
2 \\
(3 \cdot 1) \\
5 \\
(16 \cdot 1)\end{array}$} & \multirow{8}{*}{$\begin{array}{c}14^{\star \star} \\
(17 \cdot 9) \\
8^{\star} \\
(27 \cdot 6) \\
2 \\
(10 \cdot 5) \\
3 \\
(13 \cdot 6) \\
2 \\
(2 \cdot 9) \\
12^{\star \star} \\
(16 \cdot 4) \\
0 \\
(0) \\
2 \\
(2 \cdot 5)\end{array}$} & \multirow{8}{*}{$\begin{array}{c}3 \\
(3 \cdot 8) \\
0 \\
(0) \\
0 \\
(0) \\
1 \\
(4 \cdot 5) \\
1 \\
(1 \cdot 5) \\
2 \\
(2 \cdot 7) \\
0 \\
(0) \\
0 \\
(0) \\
(0)\end{array}$} & \multirow{8}{*}{$\begin{array}{c}21^{\star \star} \\
(26 \cdot 9) \\
19^{\star \star} \\
(65 \cdot 5) \\
12^{\star \star} \\
(53 \cdot 2) \\
10^{\star \star} \\
(45 \cdot 4) \\
20^{\star \star} \\
(29 \cdot 9) \\
6 \\
(8 \cdot 2) \\
0 \\
(0) \\
0 \\
(0)\end{array}$} & \multirow{8}{*}{$\begin{array}{c}15^{\star \star} \\
(19 \cdot 2) \\
7^{\star \star} \\
\left(24^{\star} \cdot 1\right) \\
5^{\star \star} \\
(26 \cdot 3) \\
3^{\star} \\
(13 \cdot 6) \\
15^{\star \star} \\
(22 \cdot 4) \\
16^{\star \star} \\
(21 \cdot 9) \\
1 \\
(1 \cdot 5) \\
0 \\
(0)\end{array}$} & \multirow{8}{*}{$\begin{array}{c}19^{\star \star} \\
(24 \cdot 4) \\
7^{\star \star} \\
(24 \cdot 1) \\
5^{\star \star} \\
(26 \cdot 3) \\
7^{\star \star} \\
(31 \cdot 6) \\
15^{\star \star} \\
(22 \cdot 4) \\
15^{\star \star} \\
(20 \cdot 5) \\
1 \\
(1 \cdot 5) \\
0 \\
(0)\end{array}$} \\
\hline 1 & Men & 29 & 31 & 10 & & & & & & & & \\
\hline 2 & Women & 19 & 28 & 8 & & & & & & & & \\
\hline 3 & Women & 22 & 33 & 9 & & & & & & & & \\
\hline 4 & Women & 67 & 33 & 12 & & & & & & & & \\
\hline 5 & Women & 73 & 35 & 15 & & & & & & & & \\
\hline \multirow[t]{2}{*}{ Control } & Women & 65 & 31 & 10 & & & & & & & & \\
\hline & Men & 31 & 36 & 8 & & & & & & & & \\
\hline
\end{tabular}

${ }^{\star} \mathrm{p}<0.05 ;{ }^{\star \star} \mathrm{p}<0.01 ;$ confectionery $v$ control workers. Numbers in parentheses are percentages.

five years in confectionery and were most pronounced during exposure in the workplace.

Table 4 shows the prevalence of acute symptoms. There was a high prevalence in all groups of confectionery workers, particularly for cough, dyspnoea, burning or dryness of the throat, and eye irritation. These prevalences were greatest in the women confectioners exposed to alcohol fumes.

VENTILATORY CAPACITY

Table 5 shows the mean across shift reductions (before-after shifts) in FVC, $\mathrm{FEV}_{1}$, $\mathrm{FEF}_{50}$, and $\mathrm{FEF}_{25}$. There were statistically significant across shift reductions in all ventilatory capacity tests, most pronounced for
$\mathrm{FEF}_{50}$ (range $4 \cdot 6-13 \cdot 0 \%$ ) and for $\mathrm{FEF}_{25}$ (range $4 \cdot 7-22 \cdot 3 \%$ ).

Table 6 shows the mean measured ventilatory capacity data in confectionery workers compared with predicted and control values. Most of the preshift values were significantly lower than expected; in particular, $\mathrm{FEF}_{50}$ and $\mathrm{FEF}_{25}$ were greately reduced. Among the group of workers in confectionery as a whole $28 \%$ had an $\mathrm{FEF}_{50}$ and $35 \%$ had an $\mathrm{FEF}_{25}$ below $80 \%$ of predicted.

ENVIRONMENTAL DUST MEASUREMENT

The mean total dust concentration in our studied confectionery mill was $31 \cdot 2$ (range $4 \cdot 1-95 \cdot 5) \mathrm{mg} / \mathrm{m}^{3}$ with a respirable fraction of

Table 4 Prevalence of acute symptoms during the work shift in confectionery workers

\begin{tabular}{|c|c|c|c|c|c|c|c|c|c|c|c|}
\hline \multirow[b]{2}{*}{ Group } & \multirow[b]{2}{*}{ Sex } & \multirow[b]{2}{*}{ No } & \multirow[b]{2}{*}{ Cough } & \multirow[b]{2}{*}{ Dyspnoea } & \multicolumn{2}{|l|}{ Throat } & \multirow{2}{*}{$\begin{array}{l}\text { Eye } \\
\text { Imitation }\end{array}$} & \multicolumn{3}{|l|}{ Nose } & \multirow[b]{2}{*}{ Headache } \\
\hline & & & & & Burning & Dryness & & Secretion & Dryness & Bleeding & \\
\hline 1 & Women & 78 & $\begin{array}{l}20 \\
(25 \cdot 5) \\
\text { NSt }\end{array}$ & $\begin{array}{l}32 \\
(41 \cdot 0) \\
\text { NS }\end{array}$ & $\begin{array}{l}30^{\star} \dagger \\
(38 \cdot 5) \\
\text { NS }\end{array}$ & $\begin{array}{l}47^{\star} \\
(60 \cdot 3) \\
\text { NS }\end{array}$ & $\begin{array}{l}40 \\
(51 \cdot 3) \\
<0.05\end{array}$ & $\begin{array}{l}11 \\
(14 \cdot 1) \\
<0.02\end{array}$ & $\begin{array}{c}37 \\
(47 \cdot 4) \\
0.01\end{array}$ & $\begin{array}{l}16^{\star} \\
(20 \cdot 5) \\
\text { NS }\end{array}$ & $\begin{array}{l}36 \ddagger \\
(46 \cdot 2) \\
\text { NS }\end{array}$ \\
\hline 1 & Men & 29 & $\begin{array}{l}13 \\
(44 \cdot 8)\end{array}$ & $\begin{array}{l}15 \\
(51 \cdot 7)\end{array}$ & $\begin{array}{c}6 \\
(20 \cdot 7)\end{array}$ & $\begin{array}{l}17 \\
(58 \cdot 6)\end{array}$ & $\begin{array}{c}9 \\
(31 \cdot 0)\end{array}$ & $\begin{array}{l}10 \\
(34 \cdot 5)\end{array}$ & $\begin{array}{c}5 \\
(17 \cdot 2)\end{array}$ & $\begin{array}{c}3 \\
(10 \cdot 3)\end{array}$ & $(41 \cdot 4)$ \\
\hline 2 & Women & 19 & $\begin{array}{c}6 \\
(31 \cdot 6)\end{array}$ & $\begin{array}{l}10 \\
(52 \cdot 6)\end{array}$ & $\begin{array}{c}13^{\star} \\
(68.4)\end{array}$ & $\begin{array}{c}15 \\
(78 \cdot 9)\end{array}$ & $\begin{array}{c}10 \\
(52 \cdot 6)\end{array}$ & $\begin{array}{c}3 \\
(15 \cdot 8)\end{array}$ & $\begin{array}{l}13 \\
(68 \cdot 4)\end{array}$ & $\begin{array}{c}8^{\star} \\
(42 \cdot 1)\end{array}$ & $\begin{array}{c}9 \\
(47 \cdot 4)\end{array}$ \\
\hline 3 & Women & 22 & $\begin{array}{c}5 \\
(22 \cdot 7)\end{array}$ & $\begin{array}{c}9 \\
(40.9)\end{array}$ & $\begin{array}{c}8 \\
(36 \cdot 4)\end{array}$ & $\begin{array}{l}18^{\star} \\
(81 \cdot 8)\end{array}$ & $\begin{array}{c}13 \\
(59 \cdot 1)\end{array}$ & $\begin{array}{c}4 \\
(18 \cdot 2)\end{array}$ & $\begin{array}{c}6 \\
(27 \cdot 3)\end{array}$ & $\begin{array}{c}6 \\
(27 \cdot 3)\end{array}$ & $(54 \cdot 5)$ \\
\hline 4 & Women & 67 & $\begin{array}{l}24 \\
(35 \cdot 8)\end{array}$ & $\begin{array}{c}30 \\
(44 \cdot 8)\end{array}$ & $\begin{array}{c}36 t \\
(53 \cdot 7)\end{array}$ & $\begin{array}{l}44 \\
(56 \cdot 7)\end{array}$ & $\begin{array}{l}37 \\
(55 \cdot 2)\end{array}$ & $\begin{array}{c}9 \\
(13 \cdot 4)\end{array}$ & $\begin{array}{l}35 \\
(52 \cdot 2)\end{array}$ & $\begin{array}{l}16 \\
(23 \cdot 9)\end{array}$ & $\begin{array}{c}45 \ddagger \\
(67 \cdot 2)\end{array}$ \\
\hline 5 & Women & 73 & $\begin{array}{l}19 \\
(26 \cdot 0)\end{array}$ & $\begin{array}{l}14 \\
(19 \cdot 2)\end{array}$ & $\begin{array}{l}27 \\
(36.9)\end{array}$ & $\begin{array}{l}28 \\
(38 \cdot 4)\end{array}$ & $\begin{array}{l}17 \\
(23 \cdot 3)\end{array}$ & $\begin{array}{c}4 \\
(5 \cdot 5)\end{array}$ & $\begin{array}{l}21 \\
(28 \cdot 8)\end{array}$ & $\begin{array}{l}6 \\
(8 \cdot 2)\end{array}$ & $\begin{array}{l}29 \\
(39 \cdot 7)\end{array}$ \\
\hline
\end{tabular}

${ }^{\star}+p<0.01$; groups significantly different from one another have the same superscripts.

†Indicates significant difference between men and women in group 1 . 
Table 5 Mean across shift (before-after shift) reductions in lung function in confectionery workers

\begin{tabular}{|c|c|c|c|c|c|c|c|c|c|c|}
\hline \multirow[b]{2}{*}{ Group } & \multirow[b]{2}{*}{ Sex } & \multirow[b]{2}{*}{ No } & \multicolumn{2}{|l|}{$F V C$} & \multicolumn{2}{|l|}{$F E V_{l}$} & \multicolumn{2}{|l|}{$F E F_{s 0}$} & \multicolumn{2}{|l|}{$F E F_{25}$} \\
\hline & & & $\%$ & p Value ${ }^{\star}$ & $\%$ & p Value & $\%$ & p Value & $\%$ & p Value \\
\hline $\begin{array}{l}1 \\
1 \\
2 \\
3 \\
4 \\
5\end{array}$ & $\begin{array}{l}\text { Women } \\
\text { Men } \\
\text { Women } \\
\text { Women } \\
\text { Women } \\
\text { Women }\end{array}$ & $\begin{array}{l}78 \\
29 \\
19 \\
22 \\
67 \\
73\end{array}$ & $\begin{array}{c}-1.6 \\
-1.4 \\
-3.7 \\
-2.2 \\
-1.7 \\
0\end{array}$ & $\begin{array}{l}<0.01 \\
<0.01 \\
<0.05 \\
<0.01 \\
<0.01 \\
\text { NS }\end{array}$ & $\begin{array}{l}-1.9 \\
-1.7 \\
-2.6 \\
-2.4 \\
-2.6 \\
0\end{array}$ & $\begin{array}{l}<0.01 \\
\text { NS } \\
\text { NS } \\
<0.05 \\
<0.01 \\
\text { NS }\end{array}$ & $\begin{array}{r}-6.1 \\
=4.6 \\
-13.0 \\
=8.8 \\
=7.3 \\
-11.5\end{array}$ & $\begin{array}{l}<0.01 \\
<0.01 \\
<0.01 \\
<0.01 \\
<0.01 \\
<0.01\end{array}$ & $\begin{array}{l}-19 \cdot 4 \\
-4 \cdot 7 \\
-4 \cdot 7 \\
-14 \cdot 7 \\
-22 \cdot 3 \\
-22 \cdot 3\end{array}$ & $\begin{array}{l}<0.01 \\
<0.01 \\
<0.01 \\
<0.01 \\
<0.01 \\
<0.01\end{array}$ \\
\hline
\end{tabular}

*Average before value significantly greater than after value.

$\%=$ per cent across shift reduction (((before-after)/before) $\times 100)$.

Table 6 Ventilatory capacity in confectionery workers in relation to predicted normal and to control values

\begin{tabular}{|c|c|c|c|c|c|c|c|c|c|c|c|}
\hline \multirow[b]{2}{*}{ Group } & \multirow[b]{2}{*}{ Sex } & \multirow[b]{2}{*}{ No } & \multirow[b]{2}{*}{ Measurement } & \multicolumn{2}{|l|}{$F V C$} & \multicolumn{2}{|l|}{$F E V_{l}$} & \multicolumn{2}{|l|}{$F E F_{s o}$} & \multicolumn{2}{|l|}{$F E F_{25}$} \\
\hline & & & & $\begin{array}{l}\text { Before- } \\
\text { Shift } \\
\text { (l) }\end{array}$ & $\begin{array}{l}\text { Before- } \\
\text { predicted } \\
\text { p Value }\end{array}$ & $\begin{array}{l}\text { Before- } \\
\text { shift } \\
\text { (l) }\end{array}$ & $\begin{array}{l}\text { Before- } \\
\text { predicted } \\
\text { p Value }\end{array}$ & $\begin{array}{l}\text { Before- } \\
\text { shift } \\
(\text { (ls) }\end{array}$ & $\begin{array}{l}\text { Before- } \\
\text { predicted } \\
\text { p Value }\end{array}$ & $\begin{array}{l}\text { Before- } \\
\text { shift } \\
\text { (I/s) }\end{array}$ & $\begin{array}{l}\text { Before- } \\
\text { predicted } \\
\text { p Value }\end{array}$ \\
\hline 1 & Women & 78 & $\begin{array}{l}\text { Measured } \\
\% \text { Control } \\
\% \text { Predicted }\end{array}$ & $\begin{array}{l}3.79(0.48) \\
96.7 \\
99.5\end{array}$ & NS & $\begin{array}{l}3.19(0.47) \\
96.2 \\
98.1\end{array}$ & NS & $\begin{array}{l}3.94(0.89) \\
80.3 \\
82.9\end{array}$ & $<0.01$ & $\begin{array}{l}2 \cdot 32(0 \cdot 95) \\
84 \cdot 8 \\
87 \cdot 9\end{array}$ & $<0.01$ \\
\hline 1 & Men & 29 & $\begin{array}{l}\text { Measured } \\
\text { \% Control } \\
\% \text { Predicted }\end{array}$ & $\begin{array}{l}5.13(0.91) \\
94.3 \\
93.9\end{array}$ & $<0.01$ & $\begin{array}{l}4.06(0.83) \\
93.4 \\
95.1\end{array}$ & NS & $\begin{array}{l}4.63(1.06) \\
73.4 \\
76.9\end{array}$ & $<0.01$ & $\begin{array}{l}2.67(1 \cdot 21) \\
80 \cdot 4 \\
83.98\end{array}$ & $<0.01$ \\
\hline 2 & Women & 19 & $\begin{array}{l}\text { Measured } \\
\% \text { Control } \\
\% \text { Predicted }\end{array}$ & $\begin{array}{l}3.78(0.48) \\
91.7 \\
93.8\end{array}$ & $<0.05$ & $\begin{array}{l}3.06(0.41) \\
94.1 \\
96.2\end{array}$ & NS & $\begin{array}{l}3.71(1 \cdot 10) \\
72.5 \\
76 \cdot 08\end{array}$ & $<0.01$ & $\begin{array}{l}2 \cdot 24(1 \cdot 04) \\
75 \cdot 1 \\
80 \cdot 97\end{array}$ & $<0.05$ \\
\hline 3 & Women & 22 & $\begin{array}{l}\text { Measured } \\
\text { \% Control } \\
\text { \% Predicted }\end{array}$ & $\begin{array}{l}3.62(0.66) \\
91.3 \\
90.3\end{array}$ & $<0.01$ & $\begin{array}{l}2.91(0.59) \\
90.3 \\
92.9\end{array}$ & $<0.05$ & $\begin{array}{l}3.64(1.38) \\
72.4 \\
76.5\end{array}$ & $<0.01$ & $\begin{array}{l}2.30(0.92) \\
83.5 \\
87.5\end{array}$ & $<0.05$ \\
\hline 4 & Women & 67 & $\begin{array}{l}\text { Measured } \\
\% \text { Control } \\
\% \text { Predicted }\end{array}$ & $\begin{array}{l}3.61(0.80) \\
92.4 \\
95.5\end{array}$ & NS & $\begin{array}{l}3.06(0.48) \\
93.2 \\
96.2\end{array}$ & NS & $\begin{array}{l}3.98(1.19) \\
80.0 \\
84.5\end{array}$ & $<0.01$ & $\begin{array}{l}2 \cdot 15(1 \cdot 00) \\
78 \cdot 3 \\
82 \cdot 4\end{array}$ & $<0.01$ \\
\hline 5 & Women & 73 & $\begin{array}{l}\text { Measured } \\
\% \text { Control } \\
\% \text { Predicted }\end{array}$ & $\begin{array}{l}3.38(0.60) \\
90.5 \\
91.6\end{array}$ & $<0.01$ & $\begin{array}{l}2.53(0.53) \\
90.5 \\
90.0\end{array}$ & $<0.01$ & $\begin{array}{l}4.00(0 \cdot 26) \\
85 \cdot 2 \\
88 \cdot 1\end{array}$ & $<0.01$ & $\begin{array}{l}1.96(1.08) \\
79.4 \\
83.8\end{array}$ & $<0.01$ \\
\hline
\end{tabular}

Ventilatory capacity data are presented as mean (SD). Measured preshift baseline measurements are compared with controls; statistical differences were the same when worker values were compared with controls.

$2 \cdot 0$ (range $0.3-5 \cdot 4) \mathrm{mg} / \mathrm{m}^{3}$. Group 1 had the highest dust exposure (total dust $30 \cdot 0-95.5$ $\mathrm{mg} / \mathrm{m}^{3}$; respirable fraction $3 \cdot 0-5 \cdot 4 \mathrm{mg} / \mathrm{m}^{3}$ ), then group 3 (total dust $20 \cdot 0-35 \cdot 6 \mathrm{mg} / \mathrm{m}^{3}$; respirable fraction $15-3 \cdot 2 \mathrm{mg} / \mathrm{m}^{3}$ ) and group 4 (total dust $4 \cdot 1-12 \cdot 4 \mathrm{mg} / \mathrm{m}^{3}$; respirable fraction $0.3-1.7 \mathrm{mg} / \mathrm{m}^{3}$ ). These values for total dust exceed the maximum allowable concentrations for organic dust (vegetable and animal) prescribed by the Croatian occupational standards (total dust $10 \mathrm{mg} / \mathrm{m}^{3}$; respirable fraction $3 \mathrm{mg} / \mathrm{m}^{3}$ ). Concentrations of ethyl alcohol vapours varied from 316 to 3421 ppm, and were also often higher than allowed by the Croatian standard (1000 ppm).

\section{Discussion}

Our data suggest that work in confectionery plants may cause the development of chronic respiratory symptoms accompanied by deterioration in lung function in many workers. These changes are particularly pronounced in those workers exposed to the dust of flour, starch, and sugar as well as in those exposed to alcohol vapours (groups 1 and 2).

Baur et $a l^{26}$ suggested that various baking components, especially mould enzymes play a significant part in the causation of baker's asthma. In their study $5 \%$ to $24 \%$ of bakers were sensitive to several carbohydrate splitting enzymes as well as to soy flour. Our previous study of soy bean workers showed that working with soy flour is associated with the development of respiratory symptoms and changes in lung function. ${ }^{27}$

Taytard et $a l^{28}$ showed that in flour mill workers there was a significantly higher prevalence of chronic cough, chronic phlegm, and airway hyper-reactivity than in control workers $(p<0.01)$ but no differences were noted in prevalences of asthma or allergy. Debelic and Sarvan found considerably higher prevalences of asthma and rhinitis (58\%) in bakers than we found in our group 1 workers (nasal catarrh: $\quad 19 \cdot 2-24 \cdot 1 \%$; asthma $3 \cdot 8 \%) .^{5}$ Recently Musk et $\mathrm{al}^{4}$ recorded that $35 \%$ of workers in a British bakery reported chest tightness and $38 \%$ complained of nasal catarrh, which is similar to the findings in our present study of confectionery workers. The same authors demonstrated that the standardised $\mathrm{FEV}_{1} / \mathrm{FVC}$ ratio tends to decrease with increasing duration of exposure. One third of their workers had measurable bronchial hyper-reactivity $\left(\mathrm{PD}_{20}<120 \mu \mathrm{mol}\right)$. DeZotti et al studied bakers, confectioners, and pizza makers and found that $25 \%$ to $26 \%$ of exposed workers complained of chronic respiratory symptoms. They also described upper respiratory symptoms such as eye and nose irritation and chest tightness with prevalences of $16.6 \%$ to $21.4 \%$ respectively. ${ }^{29}$ Jarvinen et al found that among 234 bakery workers $23 \%$ had allergic rhinitis and $9 \%$ had asthma. ${ }^{30}$ These data for rhinitis are similar to those 
obtained in our workers (nasal catarrh 13.6$26 \cdot 3 \%$ ). We found a somewhat lower prevalence of asthma, however, (range $1 \cdot 5-4.5 \%$ ), which is most likely due to a healthy worker effect. None of our seven subjects with occupational asthma had a history or signs of prior atopic disease before starting work in this confectionary.

Nuts and chocolate have often been reported as a cause of allergic reactions. Settipane described "restaurant syndrome" caused by food allergens such as peanuts, walnuts, and other foods. ${ }^{31}$ Allergy to chocolate was reported by Maslansky and Wein who found clinical symptoms of allergy in $38 \%$ of their subjects, which were reproduced after ingestion of cocoa capsules. ${ }^{32}$

Workers in confectionery are also exposed to egg powder. Langeland has reported that egg white may provoke various symptoms in hypersensitive people and may act as an allergen by inhalation as well as by ingestion. ${ }^{32}$ Edwards et al ${ }^{33}$ described the development of asthmatic symptoms in eight of 13 workers exposed to the spray of an egg solution used to coat meat balls. In our study one woman in group 1 complained of chest tightness, difficulty in breathing, and itchy skin when exposed to an egg powder.

In our study many of the confectionery workers complained of acute headache during the workshift (range 39.7-54.5\%). Similar results have been obtained in our previous studies of workers exposed to coffee. ${ }^{34}$ tea, ${ }^{35}$ soy, ${ }^{27}$ and spices. ${ }^{36}$ Frequent headache due to exposure to wheat, orange, eggs, tea, coffee, chocolate, milk, corn, cane sugar, and yeast were also reported by Grant. ${ }^{37}$ Migraine due to exposure to chocolate has been reported by Blamoutier. ${ }^{38}$

Our data indicate that working in a confectionery plant may result in acute and chronic changes in lung function. Such changes were particularly pronounced for flow rates at low lung volumes $\left(\mathrm{FEF}_{50}\right.$ and $\mathrm{FEF}_{25}$ ) suggesting obstructive changes in the smaller airways.

Prevention of the potential airway effects from occupational exposures in confectionery plants requires the environmental control of dust levels. Also, medical surveillance may identify and protect sensitive workers from developing chronic obstructive respiratory disease once respiratory symptoms begin. Lung function tests should be performed before and after the workshift by recording MEFV curves to identify sensitive workers. Those with pre-existing lung disease and smokers may be at particular risk for working in those areas of confectionery manufacture that induce respiratory allergy and acute or chronic respiratory disease.

This research was supported in part by grant No YF 733 from the National Institute of Health, Bethesda, MD, USA, grant No RO1-OHO 2593-O1A1 from the National Institute of Occupational Safety and Health, Center for Disease Control, Atlanta GA, USA, and by the Henry and Catherine Gaissman Foundation, New York, NY, USA
1 Brooks SM. The evaluation of occupational airways disease in the laboratory and workplace. $\mathcal{f}$ Allergy Clin Immunol 1982;70:65-6.

2 Chan-Yeung M, Lam S. Occupational asthma. Am Rev Respir Dis 1986;133:686-703.

3 Newman-Taylor AJ. Occupational asthma. Postgrad Med $\mathcal{F}$ 1988;64:505-10.

4 Musk AW, Venables KM, Crook B, Nunn AJ, Hawkins R, Crook GDW, et al. Respiratory symptoms, lung function, and sensitisation to flour in a British bakery. $\mathrm{Br} \mathcal{F}$ Ind Med 1989;46:636-42.

5 Debelic M, Sarvan B. Occupational flour allergy: baker's asthma and rhinitis. Praxis und Klinik der Pneumologie asthma and rhin

6 Block G, Tse KS, Kijek K, Chan H, Chan-Yeung M. Baker's asthma. Clinical Allergy 1983;13:359-70.

7 Block G, Tse KS, Kijek K, Chan H, Chan-Yeung M Baker's asthma. Clinical Allergy 1984;14:177-85.

8 Walker CL, Grammer LC, Shaughnessy MA, Patterson R. Bakers asthma: report on an unusual case. $\mathcal{F}$ Occup $M e d$ 1989;31:439-42.

9 Hartman AL, Wutrich B, Deflorin-Stolz R, Helfenstein U, Hewitt B, Guerin B. Atopie-screening: prick multitest, gesamt-IgE order RAST? Schweiz med Wochenschr 1985 115:466-75.

10 Speer F. Multiple food allergy. Ann Allergy 1987;34:71-6.

11 Fine AJ. Hypersensitivity reaction to pine nuts (Pinon nuts-Pignolia). Ann Allergy 1987;59:183-4.

12 Nielsen HN. Systemic allergic reaction to pine nuts. $A n n$ Allergy 1990;64:132-3.

13 Armentia A, Quintero A, Fernandez-Garcia A, Salvador J, Martin-Santos M. Allergy to pine pollen and pinon nuts a review of three cases. Ann Allergy 1990;64:49-53.

14 Santos IM, Unger L. Severe allergic reaction to pignolia nut. Ann Allergy 1958;16:459-61.

15 Fries JH. Peanuts: allergic and other untoward reactions. Ann Allergy 1982;48:220-6.

16 Yunginger JW, Squillance DL, Jones RT, Helm RK. Fatal anaphylactic reactions induced by peanuts. Allergy Proc 1989;10:249-53.

17 Bush RK, Clayton D. Asthma due to Central America walnut (Fuglans olanchana) dust. Clinical Allergy 1983;13 389-94.

18 Fries JH. Chocolate: a review of published reports of allergic and other deleterious effects, real or presumed. $A n n$ gic and other deleterious

19 Fries JH. The cocoa bean and the allergic child. Ann Allergy 1966;24:484-91.

20 Wuthrich B. Akut rezidivierendes Quincke-edem bei allergie auf malzextrakt. Schweiz med Wochen 1984;114:269-71.

21 Uragoda CG. Asthma and other symptoms in cinnamon workers. Br f Ind Med 1984;41:224-7.

22 British Medical Research Council Committee on the Aetiology of Chronic Bronchitis. Standardised questionnaire on respiratory symptoms. BMF 1960;2:1665.

23 World Health Organisation. Detection of occupational disease. Geneva: WHO, 1986:35-9.

24 Quanjer $\mathrm{Ph}$. Standardized lung function testing. Report of the working party on "standardization of lung function the working party on "standardization of lung function Bull Eur Physiopathol Respir 1983;19(suppl 5):1-95.

25 Ferris BG. Epidemiology standardization project. III. recommended standardized procedures for pulmonary function testing. Am Rev Respir Dis 1978;118:55-88.

26 Baur X, Weiss W, Sauer W, Fruhmann G, Kimm KW, Ulmer WT, et al. Baking components as a contributory cause of baker's asthma. Deutsche Medizinische Wochenschrift 1988;113:1275-30.

27 Zuskin E, Skuric Z, Kanceljak B, Pokrajac D, Schacter EN, Witek TJ. Respiratory symptoms and ventilatory capacity in soy bean workers. Am F Ind Med 1988;14: 157-65.

28 Taytard A, Faugere JG, Fontan J, Tessier JF, Gachie JP, Redon S, et al. Respiratory function in flour-mill workers. Eur 7 Epidemiol 1988;4:104-9.

29 DeZotti R, Patussi V, Larese F, Fiorito A, Negro C, Piscano $M$. Respiratory symptoms in bakers and confectioners. XVth Congress, European Academy of Allergy and tioners. XVth Congress, European Academy of Allergy

30 Jarvinen KAJ, Pirila V, Bjorksten F, Keskinen H, Lehtinen Jarvinen KAJ, Pirila V, Bjorksten F, Keskinen $\mathrm{H}$, Lehtinen
$\mathrm{M}$, Stubb S. Unsuitability of bakery work for a person with atopy: a study of 234 bakery workers. Ann Allergy 1979;42:192-5.

31 Settipane GA. The restaurant syndromes. New England and Regional Allergy Proceedings 1987;8:39-46.

32 Langeland T. A clinical and immunological study of allergy to hen's egg white. Clinical Allergy 1983;13:371-82.

33 Edwards JH, McConnochie K, Trotman DM, Collins G, Saunders MI, Latham SM. Allergy to inhaled egg material. Clinical Allergy 1983;13:427-32.

34 Zuskin E, Valic F, Skuric Z. Respiratory function in coffee workers. Br ₹ Ind Med 1979;36:117-22.

35 Zuskin E, Skuric $Z$. Respiratory function in tea workers. Br F Ind Med 1984;41:88-93.

36 Zuskin E, Skuric Z, Kanceljak B, Pokrajac D, Schachter EN, Witek TJ. Respiratory findings in spice factory EN, Witek TJ. Respiratory findings in spi
workers. Arch Environ Health 1988;43:335-9.

37 Grant ECG. Food allergies and migraine. Lancet 1979;i: 966-8.

38 Blamoutier V. Considerations sur les crises de migraine allergique au chocolat. Rev Francaise d'Allergologie 1966; 6:33-6. 